\title{
VVVX-Gaia discovery of a low luminosity globular cluster in the Milky Way disk
}

E. R. Garro ${ }^{1}$, D. Minniti ${ }^{1,2}$, M. Gómez ${ }^{1}$, J. Alonso-García ${ }^{3,4}$, R. H. Barbá ${ }^{5}$, B. Barbuy ${ }^{6}$, J. J. Clariá ${ }^{7}$, A. N. Chené ${ }^{\text {, }}$ B. Dias ${ }^{9}$, M. Hempel ${ }^{1}$, V. D. Ivanov ${ }^{10}$, P. W. Lucas ${ }^{11}$, D. Majaess ${ }^{12,13}$, F. Mauro ${ }^{14}$, C. Moni Bidin ${ }^{14}$, T. Palma ${ }^{7}$, J. B. Pullen ${ }^{1}$, R. K. Saito ${ }^{15}$, L. Smith ${ }^{16}$, F. Surot ${ }^{17}$, S. Ramírez Alegría ${ }^{3}$, M. Rejkuba ${ }^{11}$, V. Ripepi ${ }^{18}$, and J. Fernández Trincado ${ }^{19}$

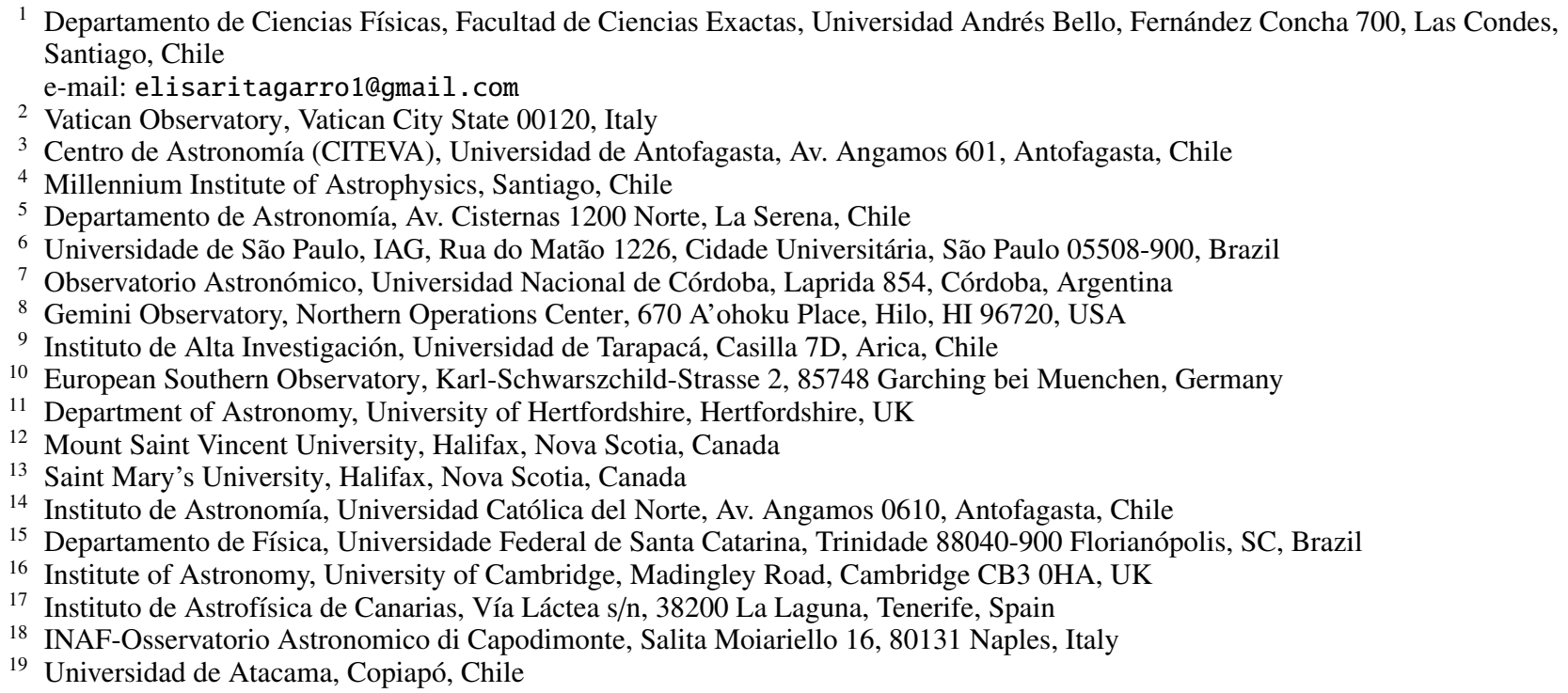

Received 21 August 2020 / Accepted 29 September 2020

\begin{abstract}
Context. Milky Way globular clusters (GCs) are difficult to identify at low Galactic latitudes because of high differential extinction and heavy star crowding. The new deep near-infrared (IR) images and photometry from the VISTA Variables in the Via Láctea Extended Survey (VVVX) allow us to chart previously unexplored regions.

Aims. Our long term aim is to complete the census of Milky Way GCs. The immediate goals are to estimate the astrophysical parameters for the newly discovered GC candidates, measuring their reddenings, extinctions, distances, total luminosities, proper motions, sizes, metallicities, and ages.

Methods. We used the near-IR VVVX survey database, in combination with the optical photometry and proper motions (PMs) from Gaia Data Release 2 (DR2), and with the Two Micron All Sky Survey photometry to search for and characterise new GCs within the southern Galactic plane $\left(|b|<5^{\circ}\right)$.

Results. We report the detection of a heretofore unknown Galactic GC at RA = 14:09:00.0; Dec $=-65: 37: 12$ (J2000) corresponding to $l=310.828 \mathrm{deg}$; and $b=-3.944 \mathrm{deg}$ in galactic coordinates. We calculate a reddening of $E\left(J-K_{s}\right)=(0.3 \pm 0.03) \mathrm{mag}$ and an extinction of $A_{K_{s}}=(0.15 \pm 0.01) \mathrm{mag}$ for this new GC. Its distance modulus and corresponding distance were measured as $(m-M)=(15.93 \pm 0.03)$ mag and $D=(15.5 \pm 1.0) \mathrm{kpc}$, respectively. We also estimate the metallicity and age by comparison with known GCs and by fitting PARSEC and Dartmouth isochrones, finding $[\mathrm{Fe} / \mathrm{H}]=(-0.70 \pm 0.2)$ dex and $t=(11.0 \pm 1.0) \mathrm{Gyr}$. The mean

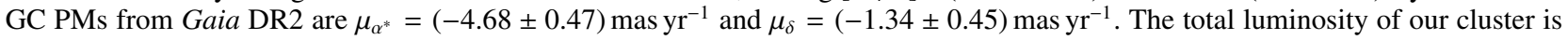
estimated to be $M_{K s}=(-7.76 \pm 0.5)$ mag. The core and tidal radii from the radial density profile are $r_{\mathrm{c}} \sim 2.1^{\prime}(4.6 \mathrm{pc})$ and $r_{\mathrm{t}}=6.5^{\prime}$ $(14.6 \mathrm{pc})$ at the cluster distance.

Conclusions. We have found a new low luminosity, old and metal-rich GC, situated in the far side of the Galactic disk at $R_{G}=11.2 \mathrm{kpc}$ from the Galactic centre and at $z=1.0 \mathrm{kpc}$ below the plane. Interestingly, the location, metallicity, and age of this GC are coincident with the Monoceros ring structure.
\end{abstract}

Key words. Galaxy: disk - Galaxy: stellar content - globular clusters: general - infrared: stars - surveys 


\section{Introduction}

While most Galactic globular clusters (GCs) have been found using optical wavelength observations, large infrared surveys have made it possible to discover many more of them in recent decades. The main surveys are the Two Micron All Sky Survey (2MASS) observed in both hemispheres with two $2 \mathrm{~m}$ telescopes, one located at the Cerro Tololo Inter-American Observatory and the other at Kitt Peak National Observatory (Skrutskie et al. 2006; Cutri et al. 2003), the Galactic Legacy Infrared Midplane Survey Extraordinaire (GLIMPSE) based on observations with the NASA/ESA Spitzer Space Telescope (Benjamin et al. 2005), the UKIDSS Galactic Plane Survey (GPS) based on observations at the UKIRT 4 m telescope in Hawaii (Lucas et al. 2008), the NASA Wide Infrared Satellite Experiment (WISE; Wright et al. 2010), and the VISTA Variables in the Via Láctea Survey based on observations at the VISTA $4 \mathrm{~m}$ telescope (VVV; Minniti et al. 2010; Saito et al. 2012). This last survey, in particular, was expanded, becoming the VISTA Variables in the Via Láctea eXtended Survey (VVVX) with observations ranging from 2017 to 2021, also using the Visual-Infrared Camera (VIRCAM) at the VISTA telescope at ESO Paranal Observatory. The VVV has already enabled the discovery and characterisation of numerous new clusters (e.g. Minniti et al. 2011,2017; Borissova et al. 2011; Moni Bidin et al. 2011; Ivanov et al. 2017; Gran et al. 2019; Camargo \& Minniti 2019), and its extension VVVX is delivering on the promise to find even more in the extended area (e.g. Borissova et al. 2018).

However, the search for new GCs is still an intricate undertaking, since many considerations about the formation and evolution of GCs have to be included in this type of study. Clusters are not likely to contribute to the stellar halo much (e.g. Martell et al. 2011; Koch et al. 2019; Reina-Campos et al. 2020), but it is known that the clusters are disrupted, especially those with an orbit going through the bulge and disk (e.g. Baumgardt \& Makino 2003; Kruijssen et al. 2011). Hence in the dense disk and bulge areas, there may be many small clusters that have lost part of their mass or had fewer stars from the beginning given the cluster initial mass function that predicts many more low mass clusters that are forming than are observed today among old GCs. Therefore, the detection of some GCs may be complicated due to their low luminosities and also the presence of dust and field stars that probably cover these objects.

\section{Discovery of Garro01}

The search and physical characterisation of Galactic GCs is one of the scientific goals of our VVVX survey, which already yields some results (Borissova et al. 2018, and in prep.; Barbá et al. 2019; Minniti et al., in prep.; Garro et al., in prep.; Obasi et al., in prep.). Here, we report on the discovery and confirmation of VVVX-GC-140900-653712 (hereafter Garro01 for short), a distant new GC deeply embedded in the Milky Way (MW) disk. Following the same procedure described by Minniti et al. (2017), this cluster was found in the VVVX database as a clear overdensity of red giant stars above the background. This GC lies in a complex low-latitude region, with variable extinction, high stellar density, containing evidence for star formation towards the Galactic plane, and also close $\left(\right.$ at $\left.\sim 1^{\circ}\right)$ in projection to the Circinus Seyfert 2 galaxy that is very extended on the sky (Freeman et al. 1977). From the first visual analysis, for example, Garro01 looks like a low luminosity GC compared with our previous finding of VVV-GC-05, which is a metalpoor $[\mathrm{Fe} / \mathrm{H}]=-1.3 \mathrm{dex} \mathrm{GC}$ located in the same region of the sky at $l=330^{\circ}, b=-1.8^{\circ}$, and $D=7.5 \mathrm{kpc}$ (Minniti et al. 2017; Contreras Ramos et al. 2018). We also observe Garro01 as a clear excess of stars above the background in the optical, where the Gaia Data Release 2 (DR2; Gaia Collaboration L19, page 2 of 8 2018a) source density map of the region (Fig. A.1) clearly shows a nearly circular region. We have visually selected it, similar to a circle with $r \sim 2.5^{\prime}$ centred at equatorial coordinates $\mathrm{RA}=14: 09: 00.0$ and $\mathrm{Dec}=-65: 37: 12(\mathrm{~J} 2000)$, corresponding to Galactic coordinates $l=310.8278^{\circ}, b=-3.9442^{\circ}$.

\section{Physical characterisation of the new GC Garro01}

We used a combination of near-infrared (IR) and optical data, obtained with the VVVX, the 2MASS, and Gaia DR2 surveys in order to measure the main parameters for Garro01. While we downloaded the available data from the respective repositories for 2MASS and Gaia DR2, we used a preliminary version of the point spread function (PSF) photometry for VVVX, which we are developing for its whole footprint based on Alonso-García et al. (2018) VVV PSF photometry. This VVVX PSF photometry was already used by Barbá et al. (2019) for the discovery and characterisation of FSR-1758, a giant metalpoor retrograde GC (Villanova et al. 2019), and by Minniti et al. (2018) for the discovery of extra tidal RR Lyrae stars in the metal-poor bulge GC NGC 6266 (M 62). For our analysis, we put the VVVX PSF photometry of our region of interest in the 2MASS $J H K_{s}$ photometric system.

We built a clean, decontaminated catalogue of highlyprobable cluster members, benefiting from the precise astrometry and proper motions (PMs) from Gaia DR2, but also matching 2MASS+Gaia and VVVX+Gaia catalogues as a means to include both brighter and fainter sources (since stars with $K s<$ $11 \mathrm{mag}$ are saturated in VVVX photometry). We first discarded all nearby stars with a parallax $>0.5$ mas. Then, we inspected the vector PM (VPM) diagram (Fig. A.2), which shows a sharp peak on top of the broad stellar background distribution, which we interpret as the cluster mean PM. These mean PMs, as measured by Gaia DR2, are as follows: $\mu_{\alpha^{*}}=(-4.68 \pm 0.47)$ mas yr$^{-1}$

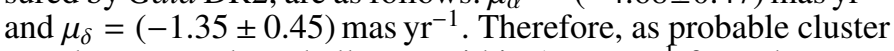
members, we selected all stars within 1 mas $^{-1} r^{-1}$ from the mean cluster PMs. Figure A.2 shows the VPM diagrams for sources with $K s<15$ mag matched in the 2MASS+Gaia catalogues and sources with $K s>13$ mag matched in the VVVX+Gaia catalogues, respectively. In the same figure, the stars located outside

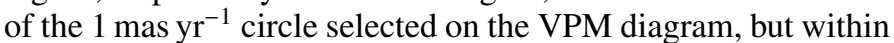
the selected radius of $2.5^{\prime}$ of the cluster centre, are shown in yellow. These stars are predominantly coming from the surrounding field that includes disk foreground and background stars.

The Gaia+2MASS colour-magnitude diagrams (CMDs) are shown in Fig. A.3, highlighting the tight red giant branch (RGB) of the PM-selected cluster members. The fact that the cluster RGB is preferentially fainter and redder than the field stars indicates that the field stars mostly belong to a foreground, less reddened population in the Galactic plane. This rules out a window in the dust distribution; as such, windows were also detected similar to stellar overdensities (e.g. Minniti 2018; Saito et al. 2020). For comparison, the fiducial RGB from the GC 47 Tuc (taken from Gaia Collaboration 2018b; Cohen et al. 2015) is overplotted in red in Gaia $G$ versus $(B P-R P)$ and in VVVX $K s$ versus $(J-K s)$, respectively; this was applied for all other colour indexes (moving it to $\Delta G=3.8 \mathrm{mag}$ and $\Delta(B P-R P)=0.39 \mathrm{mag}$ ), showing excellent agreement with the RGB of Garro01. We bring attention to the overdensity of stars around $G \sim 17.70$ in the Gaia CMD, which is well fitted with the slanted line corresponding to the location of the red clump (RC) core He burning stars in 47 Tuc.

Figure A.4 shows the luminosity functions of $G$ and $K s$ bands for a $3^{\prime}$ radius field centred on the cluster for all the red giant stars with $J-K s>0.7 \mathrm{mag}$ (top panel), as well as for the PM-selected red giants (bottom panel). These luminosity functions clearly exhibit the peaks due to the cluster RC giants at 
$G_{\mathrm{RC}}=(17.70 \pm 0.05) \mathrm{mag}$ and $K s_{\mathrm{RC}}=(14.48 \pm 0.05) \mathrm{mag}$, respectively.

The resulting PM-cleaned CMDs from the Gaia+2MASS+ VVVX data are displayed in Fig. A.5. These CMDs show that the RGB is narrow and well defined, and the cluster RC is clearly seen. It is also important to note the presence of blue star residual contamination. These stars are too bright to be blue stragglers belonging to Garro01, and we argue that this contamination arises from a fraction of Galactic foreground field stars with similar PMs as the GC.

We estimate the reddening and extinction in this field following the maps of Ruiz-Dern et al. (2018) in the near-IR, those of Schlafly \& Finkbeiner (2011) in the optical, and by taking advantage of the mean magnitude of RC giants. In detail, we assume the RC stars calibration of Ruiz-Dern et al. (2018), where the absolute magnitude in the $K s$-band is $M_{K s}=$ $(-1.601 \pm 0.009) \mathrm{mag}$ and the relative colour is $(J-K s)_{0}=$ $(0.66 \pm 0.02) \mathrm{mag}$, obtaining $E\left(J-K_{s}\right)=(0.30 \pm 0.03) \mathrm{mag}$ and $A_{K s}=(0.15 \pm 0.01) \mathrm{mag}$, respectively. In particular, we note that within $\sim 10^{\prime}$ from the GC centre, the reddening seems to be fairly uniform, with variations of $\Delta E\left(J-K_{s}\right)<0.05 \mathrm{mag}$.

The GC distance modulus can then be measured by adopting $A_{K s} / E(J-K s)=0.5 \mathrm{mag}$ (Minniti et al. 2018), which yields ( $m-$ $M)_{0}=(15.93 \pm 0.03)$ mag for Garro01, and therefore the heliocentric distance is $D=(15.5 \pm 1.0) \mathrm{kpc}$, placing this GC at $R_{G}=$ $11.2 \mathrm{kpc}$ from the Galactic centre (Bland-Hawthorn \& Gerhard 2016), assuming $R_{0}=8.3 \mathrm{kpc}$ (Dékány et al. 2013). We would like to point out that we also compared our results with other $K$-band calibration methods in order to achieve more robust parameters. We used RC magnitude theoretical calibrations from Salaris \& Girardi (2002) and Alves et al. (2002), finding a perfect agreement $\left((m-M)_{0}=15.87 \mathrm{mag}, D=15.0 \mathrm{kpc}\right.$, and $(m-M)_{0}=$ $15.93 \mathrm{mag}, D=15.3 \mathrm{kpc}$, respectively). Simultaneously, we derived the parameters using Gaia DR2 photometry. Adopting the extinction $A_{K s}=(0.15 \pm 0.01) \mathrm{mag}$, we obtain the equivalent value in the $G$-band of $A_{G}=(1.2 \pm 0.2) \mathrm{mag}$ (employing the $V$-band extinction value $A_{V}=1.4 \mathrm{mag}$ from Schlafly \& Finkbeiner 2011) and so the reddening of $E(B P-R P)=(0.39 \pm 0.06) \mathrm{mag}$. These values were used to measure the distance modulus of $(m-M)_{0}=$ $(16.0 \pm 0.2)$ mag and the heliocentric distance $D=(15.9 \pm 1.0) \mathrm{kpc}$, which is in excellent agreement with the 2MASS+VVVX photometry. The distance measurement of $D=15.5 \mathrm{kpc}$ yields a scale of $1^{\prime}=2.25 \mathrm{pc}$, placing this cluster at a height of about $1.0 \mathrm{kpc}$ below the Galactic plane.

Furthermore, we carried out the radial density profile of Garro01 in order to determine its size. First of all, we checked the central position, because an offset in its position may alter the radial density profile. Indeed, we found that the new centre is $\mathrm{RA}=212.23 \mathrm{deg}$ and Dec $=-65.628 \mathrm{deg}$ (shifted to $\Delta \mathrm{RA}=2.1^{\prime}$ and $\Delta \mathrm{Dec}=0.48^{\prime}$ from the initial coordinates). Using the resulting centre, we divided our sample into six radial bins (out to a radius of $1.5^{\prime}$ ), and we calculated the area in each bin. After that, we derived the density inside the circular annuli as the total number of stars over the area. Finally, we overplotted the King profile (King 1962) that well-reproduces density points; obtaining a core radius of $r_{\mathrm{c}}=(2.1 \pm 1.5)$ arcmin is equivalent to a physical size of $r_{\mathrm{c}}=(4.6 \pm 3.1) \mathrm{pc}$ and a tidal radius of $r_{\mathrm{t}}=6.5_{-1.9}^{+11} \mathrm{arcmin}$, corresponding to $15_{-4}^{+25} \mathrm{pc}$, which is consistent with the typical galactic GC sizes as listed in the 2010 Harris (1996) compilation. We note that the Poisson errors are very large due to a poor statistics, and the background level was supposed to be zero since the catalogue is assumed to be clean from contaminants.

The integrated GC absolute magnitude is estimated by coadding the RGB stars from the PM decontaminated diagrams and employing the GC size of 6.5 arcmin. We find $M_{K s}=$
$(-7.76 \pm 0.5) \mathrm{mag}$, which is equivalent to $M_{V}=(-5.26 \pm 1.0) \mathrm{mag}$ for typical GC mean colours of $V-K=(2.5 \pm 0.94)$ mag. Certainly, this represents a lower limit since sub-giant branch and main-sequence stars are below the observational limit. Indeed, benefiting from a strong resemblance 47 Tuc, we also estimated the total luminosity for Garro01. Briefly, we calculated the integrated absolute magnitude, coadding the RGB 47 Tuc stars and scaling to the value $M_{V}=-9.42 \mathrm{mag}$ from the 2010 Harris (1996) catalogue. This results in an absolute magnitude of $M_{V}=$ $-5.62 \mathrm{mag}$ for Garro01, placing this GC on the low luminosity tail of the MW GCLF, 2 mag fainter than the peak of the MW GCLF $\left(M_{V}=(-7.4 \pm 0.2)\right.$ mag from Harris 1991; Ashman \& Zepf 1998).

Finally, the cluster metallicity was derived following two different methods. Firstly, we compared Garro01 with the fiducial GC 47 Tuc (age $t=11.8 \mathrm{Gyr},[\mathrm{Fe} / \mathrm{H}]=-0.72 \mathrm{dex},[\alpha / \mathrm{Fe}]=$ +0.4 dex, and $D=4.5 \mathrm{kpc}$ from Brogaard et al. 2017), making reddening adjustments in order for evolutionary sequences of both GCs to coincide. Secondly, we used the fitting-isochrone method, preferring both PARSEC (Marigo et al. 2017) and Dartmouth (Dotter et al. 2008) isochrones. In order to have a good fit of the stellar isochrones in the near-IR and optical CMDs (Fig. A.5), we adopted the resulting values of reddening, extinction, and distance modulus calculated from the VVVX and Gaia photometries. Both methods provide a metallicity of $[\mathrm{Fe} / \mathrm{H}]=(-0.70 \pm 0.1) \mathrm{dex}$ and $\alpha$-enhanced between 0 and +0.4 dex. However, the cluster appears to be slightly younger than 47 Tuc because we estimate an age of $t=(11 \pm 1) \mathrm{Gyr}$ (from stellar isochrones). Briefly, the best fitting age and metallicity was obtained by iteratively fitting isochrones by comparing our data with isochrones generated with different ages and metallicities and selecting the best by-eye fit. First, we fixed the age and varied the metallicity. Then we fixed the metallicity and searched for the best fitting age. In this way, we also deduced an estimation of the metallicity and age errors, until the fittingisochrones did not reproduce all evolutionary sequences in both optical and near-IR CMDs. It is important to note that this is a rough estimation of the age since the main-sequence turn-off is below to the magnitude lower limit.

In addition to the comparison with the GC 47 Tuc, it is appropriate to make some comparisons with other known GCs. For this reason, we considered Lynga $7([\mathrm{Fe} / \mathrm{H}]=-1.01 \mathrm{dex}$, and $D=8.0 \mathrm{kpc}$, Bica et al. 2016), and NGC $5927([\mathrm{Fe} / \mathrm{H}]=$ -0.32 dex and $D=8.2 \mathrm{kpc}$, Pérez-Villegas et al. 2020), which are located in the same region of the Galactic plane, and also the $9 \mathrm{Gyr}$ old open cluster NGC $6791([\mathrm{Fe} / \mathrm{H}]=+0.3 \mathrm{dex}, D=4.1 \mathrm{kpc}$, Boesgaard et al. 2009). These comparisons do not match as well as 47 Tuc, which exhibits obvious differences in the colour of the $\mathrm{RGB}$, in particular, and also the position of the RC.

We also searched for more evidence of an old GC population, such as the RR Lyrae stars, finding two RR Lyrae candidates from the Gaia DR2 catalogue (Clementini et al. 2019) within $10^{\prime}$ of this GC. These are Gaia DR2 5851208911444645504, located 8.5 arcmin away from the GC centre at RA = 14:10:16.06 and Dec $=-65: 40: 28.6(\mathrm{~J} 2000)$, with a mean magnitude of $G=$ $16.94 \mathrm{mag}$, an amplitude of $A=0.73 \mathrm{mag}$, and a period of $P=$ 0.570059 days; and Gaia DR2 5851209426894334592, located 8.6 arcmin away at RA $=14: 10: 23.49$ and $\mathrm{Dec}=-65: 36: 56.0$ (J2000), with a mean magnitude of $G=16.33 \mathrm{mag}$, an amplitude of $A=0.94 \mathrm{mag}$, and a period of $P=0.595121$ days. Both RR Lyrae are compatible with fundamental mode pulsators (RRab type). Their distances were measured using the periodluminosity relation from Clementini et al. (2019). We believe that only Gaia DR2 5851208911444645504 may be a real cluster member, with a heliocentric distance of $D_{\mathrm{RRL}}=15.9 \mathrm{kpc}$; whereas for Gaia DR2 5851209426894334592, we calculated a 
much shorter distance of $D_{\mathrm{RRL}}=12.4 \mathrm{kpc}$. However, for both stars, their Gaia DR2 PMs of $\mu_{\alpha^{*}}=(-9.685 \pm 0.082){\text { mas } \mathrm{yr}^{-1}}^{-1}$ and $\mu_{\delta}=(-0.603 \pm 0.102)$ mas yr $^{-1}$ as well as $\mu_{\alpha^{*}}=(-5.866 \pm$ $0.085) \mathrm{mas} \mathrm{yr}^{-1}$ and $\mu_{\delta}=(-4.190 \pm 0.098) \mathrm{mas} \mathrm{yr}^{-1}$, respectively, are $>4 \sigma$ different from the mean GC PM. This result calls into question the cluster membership for both variable stars, therefore, until the RR Lyrae membership is better established with additional data, we prefer to be cautious and adopt the distance measured using the RC giants, $D=15.5 \mathrm{kpc}$. The physical parameters of the new GC Garro01 are summarised in Table A.1, along with their respective uncertainties $(1 \sigma)$.

\section{Discussion}

The discovery of this new GC demonstrates that the Galactic GC census is incomplete, so many more objects similar to this may still be found at low Galactic latitudes, hidden in very crowded, heavily reddened regions (e.g. Ivanov et al. 2000a,b, 2005; Borissova et al. 2007; Kurtev et al. 2008; Longmore et al. 2011; Valcheva et al. 2015). The known low luminosity GCs are very rare and located at high Galactic latitudes (Van Den Bergh 2003), and the low latitude ones would be particularly difficult to find.

In addition, we have to consider that the history of GCs is complex, as well as that of our Galaxy. It is known that the MW has suffered from past merging events (e.g. Ibata et al. 1994; Helmi et al. 1999, 2018; Belokurov et al. 2018; Myeong et al. 2018). Further, photometric detections of stellar streams and substructures, especially in the Galactic halo (Bell et al. 2008), are consistent with predictions from cosmological simulations that link stellar streams to accreted dwarf satellite galaxies (Bullock \& Johnston 2005). As a consequence, many GCs may be associated with these merging events (e.g. Massari et al. 2019; Vasiliev 2019).

A known structure close to the position of Garro01 is the Monoceros ring (MRi, which is also known as the Galactic anti-centre stellar structure, GASS). It was found by Newberg et al. (2002) and could represent a fitting example of a past merging event. However, the nature of MRi is still a topic of discussion. Martin et al. (2005), Peñarrubia et al. (2005), Morganson et al. (2016), and Guglielmo et al. (2017) modelled the remnant of a tidally-disrupted satellite galaxy, the Canis Major dwarf galaxy (Ibata et al. 2003; Yanny et al. 2003; Crane et al. 2003; Rocha-Pinto et al. 2003; Frinchaboy et al. 2006; Grillmair 2006; Conn et al. 2008; Sollima et al. 2011). These simulations reveal that the MRi feature should be present in the field studied here, as shown, for example, by Conn et al. (2008, see their Figs. 12 and 13). Also, spectroscopic studies have revealed that this feature is moderately metal-poor, with a mean $[\mathrm{Fe} / \mathrm{H}]=-0.8 \mathrm{dex}$ (Li et al. 2012), which is in agreement with the result presented here of $[\mathrm{Fe} / \mathrm{H}]=(-0.7 \pm$ $0.1)$ dex. However, an alternative scenario (e.g. Momany et al. 2006; Hammersley \& López-Corredoira 2011; Kalberla et al. 2014; Sheffield et al. 2018) provides support for the idea that the MRi is merely the Galactic warp and flare, composed of stars from the MW disk kicked out to their current location due to interactions between a satellite galaxy and the disk.

These results are controversial, revealing the complexity of the regions under study. This fact makes it all the more valuable to have star clusters that may be associated with the MRi structure. Indeed, Frinchaboy et al. (2006) searched for open and GCs associated with the MRi structure, concluding that Berkeley 29, Saurer 1, Tombaugh 2, Arp-Madore 2, Palomar 1, NGC 2808, and NGC 5286 were possibly associated, while $\mathrm{BH} 176$ is not. We note that the classification of $\mathrm{BH} 176$ as a
GC has been recently questioned by Sharina et al. (2014) and Vásquez et al. (2018), and it might be a unique case. On the other hand, other studies (Carraro et al. 2007; Moitinho et al. 2006) concluded that old open clusters are not consistent with the models for the MRi, which is probably just related to the Galactic warp and flare. Using more recent photometric and spectroscopic observations, Frinchaboy et al. (2014) argue that Berkeley 29 $\left([\mathrm{Fe} / \mathrm{H}]=-0.44 \mathrm{dex}, D=13.4 \mathrm{kpc}\right.$, and $\left.R_{G}=21.1 \mathrm{kpc}\right)$ and Saurer $1\left([\mathrm{Fe} / \mathrm{H}]=-0.38 \mathrm{dex}, D=13.1 \mathrm{kpc}\right.$, and $\left.R_{G}=20.2 \mathrm{kpc}\right)$ are associated with the MRi, while again BH176 is not.

Our main goal is to understand the real nature of Garro01 and derive all parameters that describe it. For this reason, we searched for a possible association to other GCs in that region, including Lynga 7, NGC 5927, 47 Tuc, BH176, and others. As mentioned in Sect. 2, we found very close similarity with 47 Tuc, so we conclude that Garro01 is a new Galactic GC. Also, given the metallicity $([\mathrm{Fe} / \mathrm{H}]=-0.7 \mathrm{dex})$ and the location ( $D=15.5 \mathrm{kpc}, R_{G}=11.2 \mathrm{kpc}$ ) of Garro01 in close proximity of the MRi structure, it is appealing to consider its possible association with the MRi.

The field star contamination, in the same field where Garro01 is located, overwhelms the GC stars, but the vast majority of the field stars along this line of sight seem to be brighter and less reddened and, therefore, are located in the foreground (Fig. A.3). They also have PMs whose mean values are different from those of the new Garro01 (Fig. A.2). Nonetheless, it is crucial to obtain radial velocities (RVs) for this cluster in order to constrain its orbital properties and to definitely confirm its association with the MRi structure. Also, we note that, according to the maps of Momany et al. (2006), there should be no warp in the specific direction of the field studied here at a Galactic longitude of $l=$ $311 \mathrm{deg}$.

Unfortunately, reliable Gaia DR2 RVs are not available for this cluster. A thorough search revealed that there is an excess of stars with RV $>200 \mathrm{~km} \mathrm{~s}^{-1}$ in this field, although all of these sources are too bright to be cluster members. Although the lack of RV measurements prevent us from calculating the orbit, the low Galactic height $(z \sim-1.1 \mathrm{kpc})$ and the very small vertical proper motion directed towards the Galac-

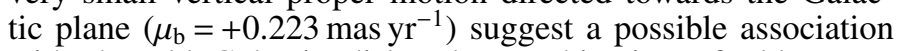
with the old Galactic disk. The combination of old age, a high metallicity, and a disk orbit closely resembles 47 Tuc and three other GCs (namely M 107, NGC 6362, and E3), whose polar paths cross in two very well-defined points on the sky (de la Fuente Marcos et al. 2015). Nevertheless, we checked that the polar path of Garro01 has no similarity with theirs, and it does not pass near these intersections. Therefore, the association of this new GC with the possible Sagittarius stream proposed by de la Fuente Marcos et al. (2015) should therefore be excluded.

\section{Conclusions}

We report the discovery of the GC Garro01 in the VVVX nearIR images. This is a low luminosity GC, located at a low latitude in the MW disk, with large reddening and high foreground stellar density. We complement our VVVX data set with 2MASS nearIR photometry as well as Gaia optical photometry and PMs.

The optical and near-IR photometry allowed us to construct CMDs in order to estimate principal GC parameters, such as age, metallicity, distance, absorption, and reddening. Most field stars are less reddened and therefore located in the foreground disk.

The optical and near-IR CMDs show that this new GC is more metal-poor and older than the canonical old metalrich open cluster NGC $6791(D=4.1 \mathrm{kpc}, t=9 \mathrm{Gyr}$, and $[\mathrm{Fe} / \mathrm{H}]=0.3$ dex, Boesgaard et al. 2009). On the other hand, we find a close similarity with the physical parameters of 
E. R. Garro et al.: VVVX-Gaia discovery of a low luminosity globular cluster in the Milky Way disk

47 Tuc $(D=4.5 \mathrm{kpc}, t=11.8 \mathrm{Gyr}$, and $[\mathrm{Fe} / \mathrm{H}]=-0.7 \mathrm{dex}$, Brogaard et al. 2017), arguing for an old GC at $D=15.5 \mathrm{kpc}$. However, this cluster is intrinsically 4.16 mag fainter than 47 Tuc, implying that its total mass is about $M \approx 10^{4} M_{\odot}$ (if 47 Tuc has $M=7 \times 10^{5} M_{\odot}$, Marks \& Kroupa 2010), on the low mass end of the Galactic GCs (Baumgardt et al. 2019). We also note the presence of two candidate RR Lyrae stars within 10 arcmin from the cluster centre. One of them has a distance consistent with the measured RC distance for the cluster. Its PMs, however, suggest that both RR Lyrae are not cluster members.

The Galactic GC census is incomplete at low latitudes (Ivanov et al. 2005; Minniti et al. 2017). Furthermore, the discovery of Garro01 suggests that more low luminosity GCs may still be uncovered at low Galactic latitudes.

Another interesting implication is that the location, distance, and metallicity of this GC match those of the MRi structure, a potential accretion event recently identified in the Galactic plane. The possibility of a physical association with this structure must be confirmed with follow-up spectroscopy to measure radial velocities and detailed chemical abundances.

Acknowledgements. ERG acknowledges support from an UNAB PhD scholarship. J.A.-G. acknowledges support from Fondecyt Regular 1201490 and from ANID, Millennium Science Initiative ICN12_009, awarded to the Millennium Institute of Astrophysics (MAS). DM and MG are supported by Proyecto FONDECYT Regular No. 1170121. DM is also supported by the BASAL Center for Astrophysics and Associated Technologies (CATA) through grant AFB 170002. We gratefully acknowledge the use of data from the ESO Public Survey program IDs 179.B-2002 and 198.B-2004 taken with the VISTA telescope and data products from the Cambridge Astronomical Survey Unit. PWL acknowledges support by STFC Consolidated Grant ST/R00905/1. SRA acknowledges support from the FONDECYT Iniciacion project 11171025 , the FONDECYT Regular project 1201490, and the CONICYT + PAI "Concurso Nacional Insercion de Capital Humano Avanzado en la Academia 2017" project PAI 79170089. R.K.S. acknowledges support from CNPq/Brazil through project 305902/2019-9.

\section{References}

Alonso-García, J., Saito, R. K., Hempel, M., et al. 2018, A\&A, 619, A4 Alves, D. R., Rejkuba, M., Minniti, D., \& Cook, K. H. 2002, ApJ, 573, L51 Ashman, K. M., \& Zepf, S. E. 1998, Cambridge Astrophysics Series (Cambridge: Cambridge University Press), 30

Barbá, R. H., Minniti, D., Geisler, D., et al. 2019, ApJ, 870, L24

Baumgardt, H., \& Makino, J. 2003, MNRAS, 340, 227

Baumgardt, H., Hilker, M., Sollima, A., \& Bellini, A. 2019, MNRAS, 482, 5138 Bell, E. F., Zucker, D. B., Belokurov, V., et al. 2008, ApJ, 680, 295

Belokurov, V., Erkal, D., Evans, N. W., Koposov, S. E., \& Deason, A. J. 2018, MNRAS, 478, 611

Benjamin, R. A., Churchwell, E., Babler, B. L., et al. 2005, ApJ, 630, L149

Bica, E., Ortolani, S., \& Barbuy, B. 2016, PASA, 33, e028

Bland-Hawthorn, J., \& Gerhard, O. 2016, ARA\&A, 54, 529

Boesgaard, A. M., Jensen, E. E. C., \& Deliyannis, C. P. 2009, AJ, 137, 4949

Borissova, J., Ivanov, V. D., Stephens, A. W., et al. 2007, A\&A, 474, 121

Borissova, J., Bonatto, C., Kurtev, R., et al. 2011, A\&A, 532, A131

Borissova, J., Ivanov, V. D., Lucas, P. W., et al. 2018, MNRAS, 481, 3902

Brogaard, K., VandenBerg, D. A., Bedin, L. R., et al. 2017, MNRAS, 468, 645

Bullock, J. S., \& Johnston, K. V. 2005, ApJ, 635, 931

Camargo, D., \& Minniti, D. 2019, MNRAS, 484, L90

Carraro, G., Moitinho, A., Zoccali, M., Vázquez, R. A., \& Baume, G. 2007, AJ, 133,1058

Clementini, G., Ripepi, V., Molinaro, R., et al. 2019, A\&A, 622, A60

Cohen, R. E., Hempel, M., Mauro, F., et al. 2015, AJ, 150, 176

Conn, B. C., Lane, R. R., Lewis, G. F., et al. 2008, MNRAS, 390, 1388

Contreras Ramos, R., Minniti, D., Gran, F., et al. 2018, ApJ, 863, 79

Crane, J. D., Majewski, S. R., Rocha-Pinto, H. J., et al. 2003, ApJ, 594, L119

Cutri, R. M., Skrutskie, M. F., van Dyk, S., et al. 2003, VizieR Online Data Catalog: II/246

de la Fuente Marcos, R., de la Fuente Marcos, C., Moni Bidin, C., Ortolani, S., \& Carraro, G. 2015, A\&A, 581, A13

Dékány, I., Minniti, D., Catelan, M., et al. 2013, ApJ, 776, L19

Dotter, A., Chaboyer, B., Jevremović, D., et al. 2008, ApJS, 178, 89
Freeman, K. C., Karlsson, B., Lynga, G., et al. 1977, A\&A, 55, 445

Frinchaboy, P. M., Muñoz, R. R., Phelps, R. L., Majewski, S. R., \& Kunkel, W. E. 2006, AJ, 131, 922

Frinchaboy, P. M., Thompson, B. A., O’Connell, J., et al. 2014, Am. Astron. Soc. Meet. Abstr. , 223, 442.12

Gaia Collaboration (Brown, A. G. A., et al.) 2018a, A\&A, 616, A1

Gaia Collaboration (Babusiaux, C., et al.) 2018b, A\&A, 616, A10

Gran, F., Zoccali, M., Contreras Ramos, R., et al. 2019, A\&A, 628, A45

Grillmair, C. J. 2006, ApJ, 651, L29

Guglielmo, M., Lane, R. R., Conn, B. C., et al. 2017, MNRAS, 474, 4584

Hammersley, P. L., \& López-Corredoira, M. 2011, A\&A, 527, A6

Harris, W. E. 1991, ARA\&A, 29, 543

Harris, W. E. 1996, AJ, 112, 1487

Helmi, A., White, S. D. M., de Zeeuw, P. T., \& Zhao, H. 1999, Nature, 402, 53

Helmi, A., Babusiaux, C., Koppelman, H. H., et al. 2018, Nature, 563, 85

Ibata, R. A., Gilmore, G., \& Irwin, M. J. 1994, Nature, 370, 194

Ibata, R. A., Irwin, M. J., Lewis, G. F., Ferguson, A. M. N., \& Tanvir, N. 2003, MNRAS, 340, L21

Ivanov, V. D., Borissova, J., \& Vanzi, L. 2000a, A\&A, 362, L1

Ivanov, V. D., Borissova, J., Alonso-Herrero, A., \& Russeva, T. 2000b, AJ, 119, 2274

Ivanov, V. D., Kurtev, R., \& Borissova, J. 2005, A\&A, 442, 195

Ivanov, V. D., Piatti, A. E., Beamín, J.-C., et al. 2017, A\&A, 600, A112

Kalberla, P. M. W., Kerp, J., Dedes, L., \& Haud, U. 2014, ApJ, 794, 90

King, I. 1962, AJ, 67, 471

Koch, A., Grebel, E. K., \& Martell, S. L. 2019, A\&A, 625, A75

Kruijssen, J. M. D., Pelupessy, F. I., Lamers, H. J. G. L. M., Portegies Zwart, S F., \& Icke, V. 2011, MNRAS, 414, 1339

Kurtev, R., Ivanov, V. D., Borissova, J., \& Ortolani, S. 2008, A\&A, 489, 583

Li, J., Newberg, H. J., Carlin, J. L., et al. 2012, ApJ, 757, 151

Longmore, A. J., Kurtev, R., Lucas, P. W., et al. 2011, MNRAS, 416, 465

Lucas, P. W., Hoare, M. G., Longmore, A., et al. 2008, MNRAS, 391, 136

Marigo, P., Girardi, L., Bressan, A., et al. 2017, ApJ, 835, 77

Marks, M., \& Kroupa, P. 2010, MNRAS, 406, 2000

Martell, S. L., Smolinski, J. P., Beers, T. C., \& Grebel, E. K. 2011, A\&A, 534, A136

Martin, N. F., Ibata, R. A., Conn, B. C., et al. 2005, MNRAS, 362, 906

Massari, D., Koppelman, H. H., \& Helmi, A. 2019, A\&A, 630, L4

Minniti, D. 2018, in The Vatican Observatory, Castel Gandolfo: 80th Anniversary Celebration, eds. G. Gionti, \& J. B. Kikwaya Eluo, 51, 63

Minniti, D., Lucas, P. W., Emerson, J. P., et al. 2010, New Astron., 15, 433

Minniti, D., Hempel, M., Toledo, I., et al. 2011, A\&A, 527, A81

Minniti, D., Palma, T., Dékány, I., et al. 2017, ApJ, 838, L14

Minniti, D., Saito, R. K., Gonzalez, O. A., et al. 2018, A\&A, 616, A26

Moitinho, A., Vázquez, R. A., Carraro, G., et al. 2006, MNRAS, 368, L77

Momany, Y., Zaggia, S., Gilmore, G., et al. 2006, A\&A, 451, 515

Moni Bidin, C., Mauro, F., Geisler, D., et al. 2011, A\&A, 535, A33

Morganson, E., Conn, B., Rix, H.-W., et al. 2016, ApJ, 825, 140

Myeong, G. C., Evans, N. W., Belokurov, V., Sanders, J. L., \& Koposov, S. E. 2018, ApJ, 856, L26

Newberg, H. J., Yanny, B., Rockosi, C., et al. 2002, ApJ, 569, 245

Peñarrubia, J., Martínez-Delgado, D., Rix, H. W., et al. 2005, ApJ, 626, 128

Pérez-Villegas, A., Barbuy, B., Kerber, L. R. O., et al. 2020, MNRAS, 491, 3251

Reina-Campos, M., Hughes, M. E., Kruijssen, J. M. D., et al. 2020, MNRAS, 493, 3422

Rocha-Pinto, H. J., Majewski, S. R., Skrutskie, M. F., \& Crane, J. D. 2003, ApJ, 594, L115

Ruiz-Dern, L., Babusiaux, C., Arenou, F., Turon, C., \& Lallement, R. 2018, A\&A, 609, A116

Saito, R. K., Hempel, M., Minniti, D., et al. 2012, A\&A, 537, A107

Saito, R. K., Minniti, D., Benjamin, R. A., et al. 2020, MNRAS, 494, L32

Salaris, M., \& Girardi, L. 2002, MNRAS, 337, 332

Schlafly, E. F., \& Finkbeiner, D. P. 2011, ApJ, 737, 103

Sharina, M. E., Donzelli, C. J., Davoust, E., Shimansky, V. V., \& Charbonnel, C. 2014, A\&A, 570, A48

Sheffield, A. A., Price-Whelan, A. M., Tzanidakis, A., et al. 2018, ApJ, 854, 47

Skrutskie, M. F., Cutri, R. M., Stiening, R., et al. 2006, AJ, 131, 1163

Sollima, A., Martínez-Delgado, D., Valls-Gabaud, D., \& Peñarrubia, J. 2011, ApJ, 726, 47

Valcheva, A. T., Ovcharov, E. P., Lalova, A. D., et al. 2015, MNRAS, 446, 730

Van Den Bergh, S. 2003, ApJ, 590, 797

Vasiliev, E. 2019, MNRAS, 484, 2832

Vásquez, S., Saviane, I., Held, E. V., et al. 2018, A\&A, 619, A13

Villanova, S., Monaco, L., Geisler, D., et al. 2019, ApJ, 882, 174

Wright, E. L., Eisenhardt, P. R. M., Mainzer, A. K., et al. 2010, AJ, 140, 1868

Yanny, B., Newberg, H. J., Grebel, E. K., et al. 2003, ApJ, 588, 824 


\section{Appendix A: Figures and table}
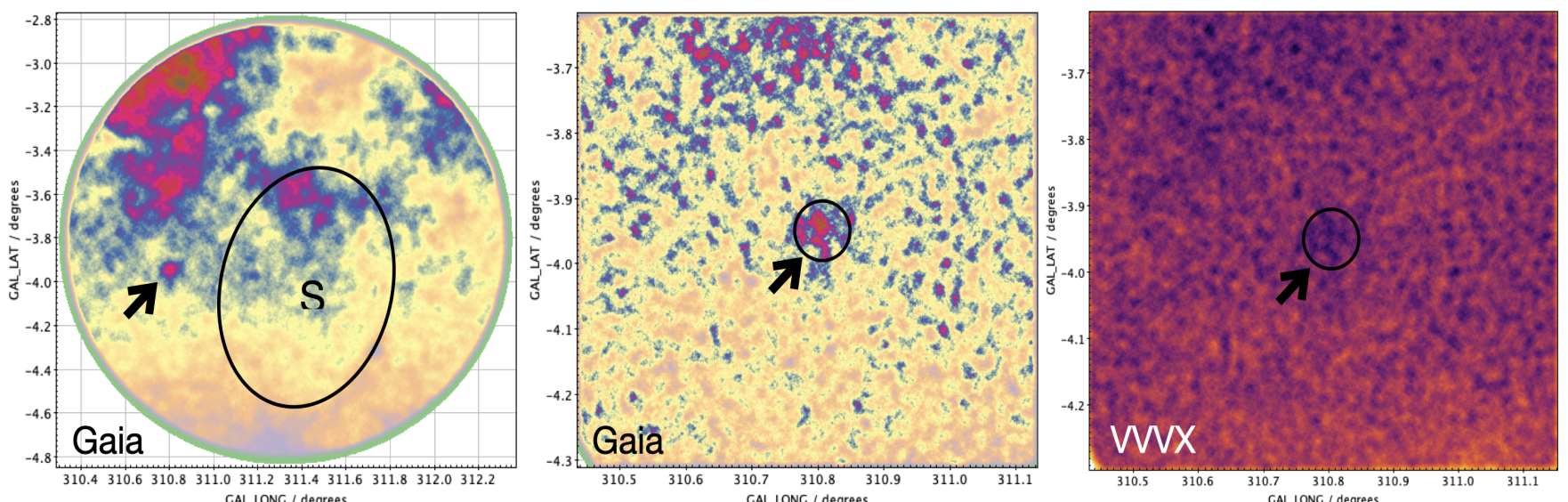

Fig. A.1. Left panel: Gaia stellar density map for a $r=2$ degree field in Galactic coordinates, indicating the new GC with an arrow, along with the size of the HI emission of the Circinus galaxy (Freeman et al. 1977). Middle and right panel: density maps of the zoomed $42^{\prime} \times 42^{\prime}$ region around Garro01 using optical Gaia data and VVVX near-IR data, respectively, where the circle indicates the approximate cluster size. It is important to note that the redder areas are representative of overdensities, while the yellower areas are lower densities.
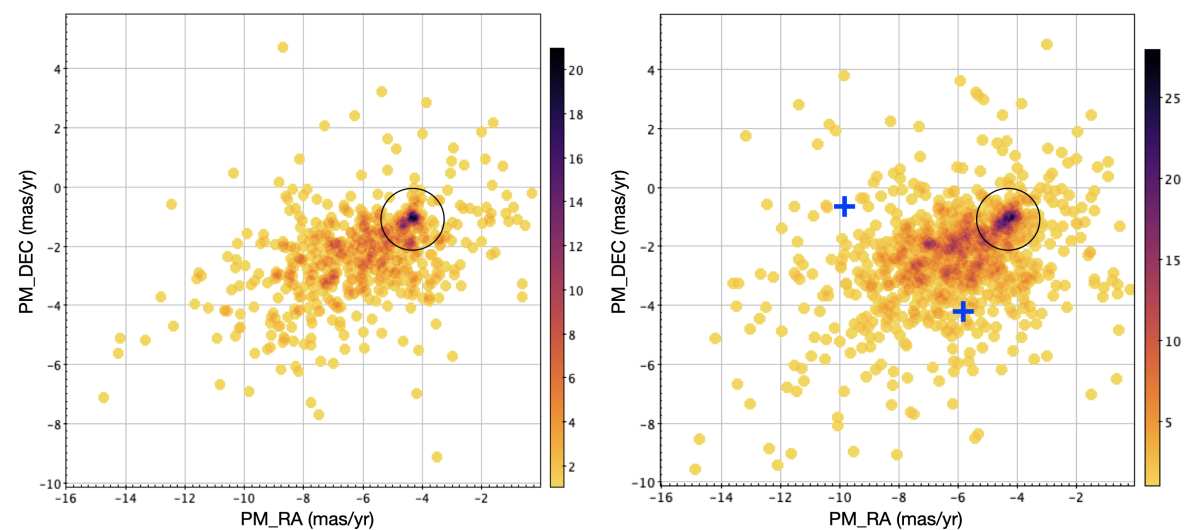

Fig. A.2. Vector PM diagrams for the bright sources $(K s<15 \mathrm{mag})$ matched in the 2MASS+Gaia catalogues (right panel), and the fainter sources $(K s>13 \mathrm{mag}$ ) matched in the VVVX+Gaia catalogues (left panel). The black circle indicates the cluster selection, while blue crosses represent the position of the two RR Lyrae found within 10' of the cluster centre. The colour bars indicate a higher (towards the black colour) and lower (towards the yellow colour) concentration. 
E. R. Garro et al.: VVVX-Gaia discovery of a low luminosity globular cluster in the Milky Way disk
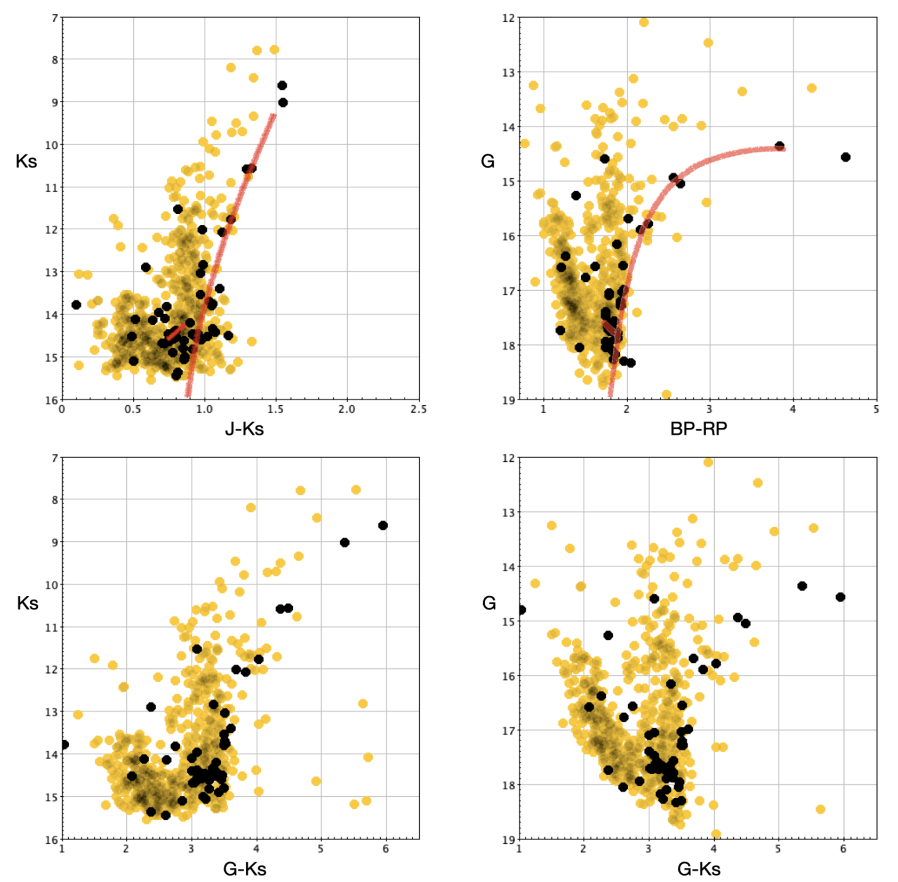

Fig. A.3. Optical and near-IR CMDs for the GC Garro01 field from 2MASS (black points) and Gaia or VVVX (yellow points), showing the PM-selected cluster members. We note that the well-defined cluster RGB located lower and to the red side of the diagrams, indicating that the fiducial RGB from GC 47 Tuc from Cohen et al. (2015) and Gaia Collaboration (2018b) is overplotted in the top panels, is appropriately shifted to the cluster distance and reddening; this is a distant and reddened cluster.
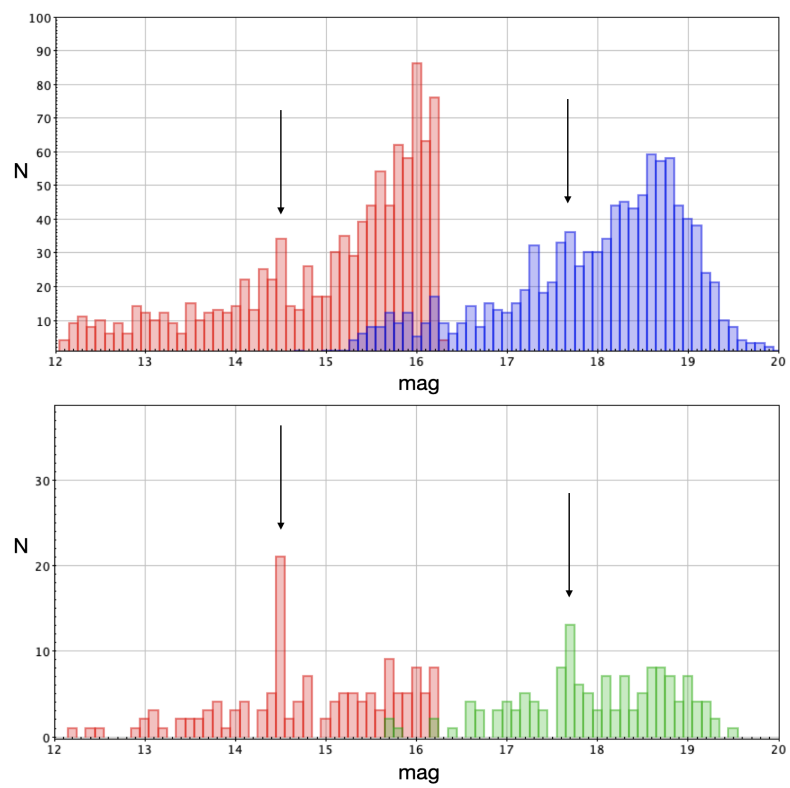

Fig. A.4. Top panel: luminosity functions for GC Garro01, showing the VVVX Ks-band photometry in red on the left, and the Gaia DR2 G-band photometry in blue on the right. The arrows point to the location of the RC. Bottom panel: same diagram showing only the counts of PM-selected sources. 

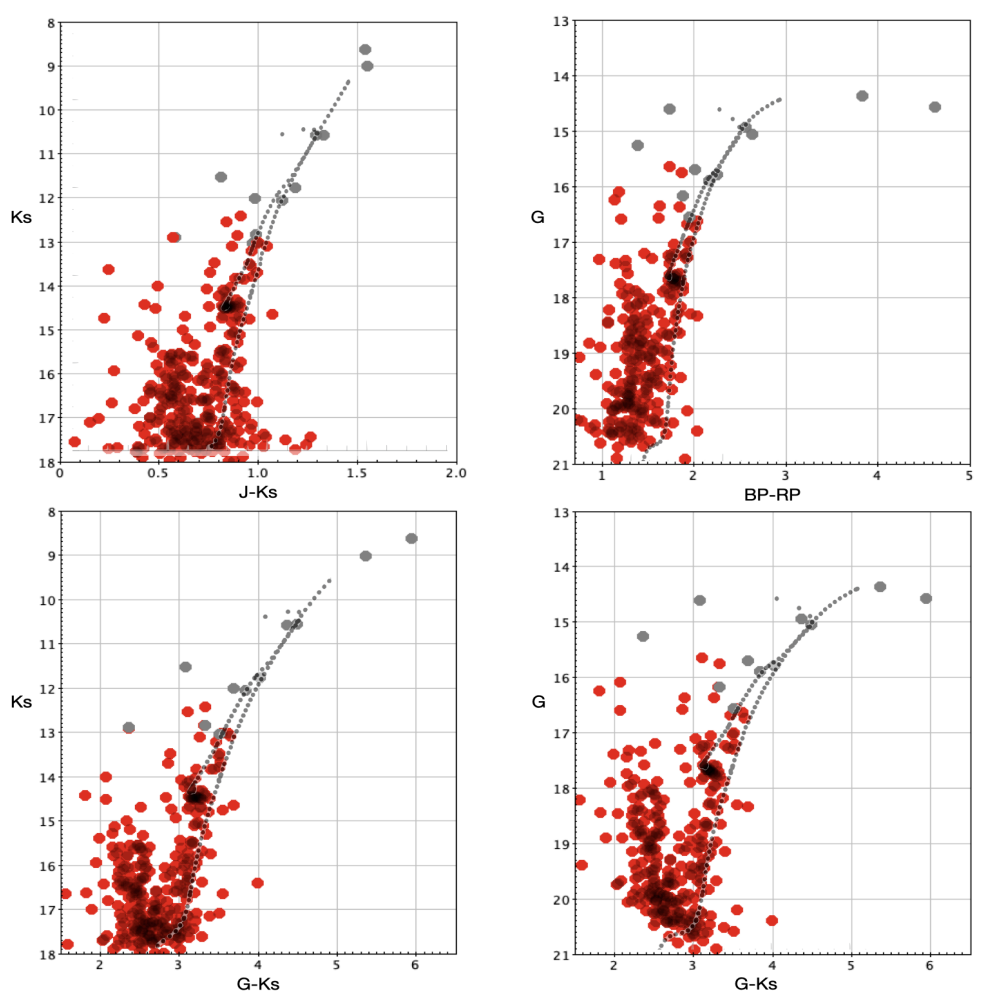

Fig. A.5. PM decontaminated CMDs for the GC Garro01, showing the VVVX near-IR CMD (top left), the Gaia DR2 optical CMD (top right), and the Gaia-VVVX optical-IR CMDs (bottom panels). Red points are VVVX-Gaia matched sources, while grey points are 2MASS-Gaia matched sources. The black dotted line shows the fit to a PARSEC isochrone with a metallicity of $[\mathrm{Fe} / \mathrm{H}]=-0.70$ dex and an age of $t=11.0 \mathrm{Gyr}$. We note that the well defined cluster RGB and RC are present in all of the CMDs, as is also the case for the residual field contamination on the blue side arising from a fraction of Galactic foreground field stars with similar PMs as the GC.

Table A.1. Final GC physical parameters.

\begin{tabular}{lc}
\hline \hline Parameter & Value \\
\hline RA $(\mathrm{J} 2000)$ & $14: 09: 00.0$ \\
Dec $(\mathrm{J} 2000)$ & $-65: 37: 12$ \\
Latitude & $310.828^{\circ}$ \\
Longitude & $-3.944^{\circ}$ \\
$\mu_{\alpha^{*}}\left[\mathrm{mas} \mathrm{yr}^{-1}\right]$ & $-4.68 \pm 0.47$ \\
$\mu_{\delta}\left[\mathrm{mas} \mathrm{yr}^{-1}\right]$ & $-1.35 \pm 0.45$ \\
$A_{K_{s}}[\mathrm{mag}]$ & $0.15 \pm 0.01$ \\
$E\left(J-K_{s}\right)[\mathrm{mag}]$ & $0.30 \pm 0.03$ \\
$(m-M)_{0}[\mathrm{mag}]$ & $15.93 \pm 0.03$ \\
$D[\mathrm{kpc}]$ & $15.5 \pm 1.0$ \\
$R_{G}[\mathrm{kpc}]$ & $11.2 \pm 0.2$ \\
$\mathrm{Height} z[\mathrm{kpc}]$ & 1.0 \\
$M_{K_{s}}[\mathrm{mag}]$ & $-7.76 \pm 0.5$ \\
$M_{V}[\mathrm{mag}]$ & $-5.26 \pm 1.0$ \\
{$[\mathrm{Fe} / \mathrm{H}][\mathrm{dex}]$} & $-0.7 \pm 0.2$ \\
Age $[\mathrm{Gyr}]$ & $11.0 \pm 1.0$ \\
$r_{\mathrm{c}}[\mathrm{arcmin}]$ & $2.1 \pm 1.5(4.6 \pm 3.1 \mathrm{pc})$ \\
$r_{\mathrm{t}}[\mathrm{arcmin}]$ & $6.5_{-1.9}^{+11}\left(15_{-4}^{+25} \mathrm{pc}\right)$ \\
\hline \multicolumn{2}{c}{} \\
\hline
\end{tabular}

